\title{
A GPU-based Pencil Beam Algorithm for Dose Calculations in Proton Radiation Therapy
}

\author{
Georgios Kalantzis \\ Department of Physics, Florida Atlantic University, 777 Glades Rd, \\ Boca Raton, FL 33431, USA \\ E-mail: gkalan@gmail.com \\ Theodora Leventouri \\ Department of Physics, Florida Atlantic University, 777 Glades Rd, \\ Boca Raton, FL 33431, USA \\ E-mail: leventou@fau.edu \\ Hidenobu Tachibana \\ Department of Radiation Oncology, National Cancer Center Hospital East, \\ 6-5-1 Kashiwanoha, Kashiwa, Chiba, 277-8577, Japan \\ E-mail: gogo.for.happy.hope.12@gmail.com \\ Charles Shang \\ Department of Radiation Oncology, Lynn Cancer Institute, 800 Meadows Rd, \\ Boca Raton, FL, 33486, USA \\ E-mail:CShang@brrh.com
}

\begin{abstract}
Recent developments in radiation therapy have been focused on applications of charged particles, especially protons. Proton therapy can allow higher dose conformality compared to conventional radiation therapy. Over the years several dose calculation methods have been proposed in proton therapy. A common characteristic of all these methods is their extensive computational burden. One way to ameliorate that issue is the parallelization of the algorithm. In the current study a GPU-based pencil beam algorithm for dose calculations with protons is proposed. The studies indicated a max speedup factor of $\sim 127$ in a homogeneous phantom.
\end{abstract}

Keywords: pencil-beam, proton therapy, GPU, dose calculations.

\section{Introduction}

Radiation therapy uses high-energy radiation to shrink tumors and kill cancer cells by damaging their DNA. Contemporary radiation therapy often involves the process where radiation beams are delivered to the cancer site from external beams. In a clinical setup, external radiation therapy most commonly involves high energy photons or electrons. However, in 1946 Wilson demonstrated the potential clinical benefits of protons for cancer treatment [1] and the first clinical evaluations of external radiation therapy with protons appeared by Lawrence et al. [2]. The dosimetric properties that establish protons attractive for radiation therapy is their nearly straight line trajectories in the matter and a narrow Bragg peak which results in insignificant dose downstream the treatment sites. That implies increased local tumor control while sparing normal tissue [3].

Dose calculations play a crucial role in proton therapy treatment. In general, the gold standard for radiotherapy dose calculations is Monte Carlo (MC) methods. However, MC simulations for charged particles is a time-consuming and computational demanding task. In the time-critical clinical environment, fast dose calculations ensure a smooth workflow, and also offer planners the opportunity to fine tune the treatment planning for each individual patient. The necessity of fast dose calculations becomes 
more apparent in intensity-modulated proton therapy [4, 5], and 4D treatment planning [6,7]. One way to ameliorate that issue is to parallelize the computational method.

Recent developments, have demonstrated the potential of Graphics processing Units (GPUs) for MC simulations for proton therapy. In particular, Kohno et al. [8] developed a simplified MC method (SMC) for proton transportation on a GPU platform. The SMC was compatible with GPU's SIMD structure in the sense that each GPU thread calculated the transportation of the proton while all of the threads performed the same instructions on different data depending on the current proton status. Track-repeating methods have also been utilized in the past for fast GPU-based MC simulations $[9,10]$. In these methods, a database of proton transport histories is generated in advance for a homogeneous water phantom using an accurate MC code. Then, for a patient case, the track-repeating $\mathrm{MC}$ calculates dose distributions by repeating proton tracks from the database and scaling the step length and scattering angle within each track according to the tissue densities.

An alternative method is the pencil beam algorithms (PBAs) for dose calculations in radiation therapy. This method has been widely utilized in daily clinical applications due to its simplicity of calculation scheme and acceptable accuracy in many clinical cases. In PBAs a broad beam is divided into a number of small rectangular (beamlets), and the dose contribution of each beamlet to every voxel is based on analytical or empirical calculations. PBAs are well suited for parallelization on GPUs since each thread can calculate the dose for each beamlet. Gu et al. [11] have reported 200-400 speedup factors for a GPU-based pencil beam method for intensity modulation radiation therapy, while Fujimoto el al. [12] reported speedups up to 20 for proton calculations.

Foca, a Matlab-based treatment planning software, was recently presented for proton radiotherapy [13]. The aforementioned software was designed primarily for research and educational purposes. In Foca, dose calculations for both, active (pencil-beam scanning) and passive (double scattering) modalities have been implemented. The main advantages of Matlab are its intuitive higher-level syntax, advanced visualization capabilities and the availability of toolboxes with several numerical methods.

In the current study we present for the first time, to our best knowledge, a GPU-based PBA for proton dose calculations in Matlab. The evaluation of our method was established on the speedup factors for square radiation fields in homogenous phantom of water.

\section{Pencil-beam algorithm for protons}

\subsection{Depth dose distributions}

In the current study we employed an analytical expression for the protons depth dose distribution [14] For a monoenergetic proton beam along the $\mathrm{z}$ axis, impinging on a homogeneous medium at $\mathrm{z}=0$ the energy fluence, $\Psi$, at depth $\mathrm{z}$ in the medium can be written in the form:

$$
\Psi(z)=\Phi(z) \cdot E(z)
$$

$\Phi(z)$ is the particle fluence, i.e. the number of protons per $\mathrm{cm}^{2}$, and $E(z)$ is the remaining energy at depth $z$. The total dose, $\mathrm{D}(\mathrm{z})$ is given by:

$$
D(z)=-\frac{1}{\rho}\left(\Phi(z) \frac{d E(z)}{d z}+\gamma \frac{d \Phi(z)}{d z} E(z)\right)
$$

where, $\gamma$ is the fraction of the energy released in the inelastic nuclear interactions and absorbed locally, and $\rho$ is the mass density of the medium. In order to determine this depth-dose curve, we only need to know the functions $\mathrm{E}(\mathrm{z})$ and $\Phi(\mathrm{z})$. The relationship between the initial energy $\mathrm{E}(\mathrm{z}=0)=\mathrm{E}_{0}$ and the range $\mathrm{z}=\mathrm{R}_{0}$ in the medium is approximately given by:

$$
R_{0}=a E_{0}^{p}
$$

With $\mathrm{p}=1.5$, this relationship is known as Geiger's rule, which is valid for protons with energies up to about $10 \mathrm{MeV}$. For higher energies used in radiation therapy the exponent $p$ increases to 1.8 . The factor $\alpha$ is approximately proportional to the square root of the effective atomic mass of the absorbing medium. It should be noted that this simplified range-energy relationship is valid in good approximation for arbitrary media and various particles with atomic masses approximately between one (protons) and twelve (carbon ions) [15]. The beam deposits energy in the medium along its path from $\mathrm{z}=0$ to $\mathrm{z}=\mathrm{R}_{0}$. Thus, according to the range-energy relationship:

$$
E(z)=\frac{1}{a^{1 / p}}\left(R_{0}-z\right)^{1 / p}
$$

Lee et al. [16] have derived the proportionality for the protons fluence:

$$
\Phi(z) \propto \frac{1}{1-P\left(R_{0}-z\right)}
$$

Where $\mathrm{P}$ is the probability that protons may be lost from the beam due to nuclear interactions. By normalizing to the initial fluence $\Phi_{0}$ we obtain: 


$$
\Phi(z)=\Phi_{0} \frac{1+\beta\left(R_{0}-z\right)}{1+\beta R_{0}}
$$

Finally, the depth-dose distribution can be calculated as follows for $\mathrm{z}<\mathrm{R}_{0}$ :

$$
D(z)=\Phi_{0} \frac{\left(R_{0}-z\right)^{1 / p-1}+(\beta+\gamma \beta p)\left(R_{0}-z\right)^{1 / p}}{\rho p a^{1 / p}\left(1+\beta R_{0}\right)}
$$

where the slope parameter $\beta$ was determined to be $\beta=0.012 \mathrm{~cm}^{-1}$. Expression (7) gives D in units of $\mathrm{MeV} / \mathrm{g}$, if $\rho$ is given in $\mathrm{g} / \mathrm{cm}^{3}$. To obtain D in Gy, one needs to multiply by the factor $10^{9} \mathrm{e} / \mathrm{C}=1.602 \times 10^{-10}$, where e is the elementary charge.

Fig. 1 illustrates the depth-dose distributions in water for protons with initial energy $50 \mathrm{MeV}$ (solid line) and $150 \mathrm{MeV}$ (dashed line) respectively. Similarly, Fig. 2 shows the proton energy as a function of depth in water for initial energy $50 \mathrm{MeV}$ (solid line) and 150 $\mathrm{MeV}$ (dashed line) respectively.

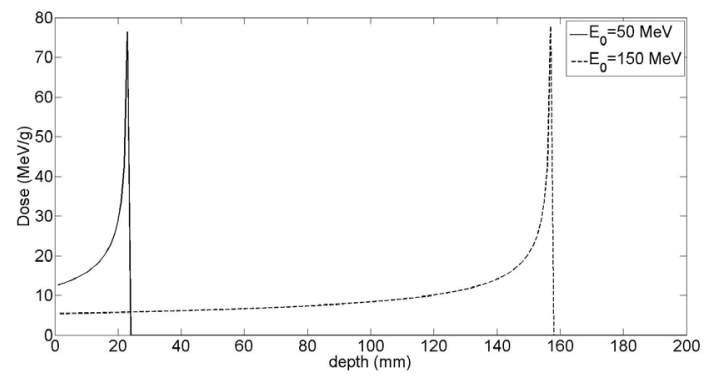

Fig 1. Depth-dose distribution for $50 \mathrm{MeV}$ (solid line) and 150 $\mathrm{MeV}$ (dashed line) protons in water.

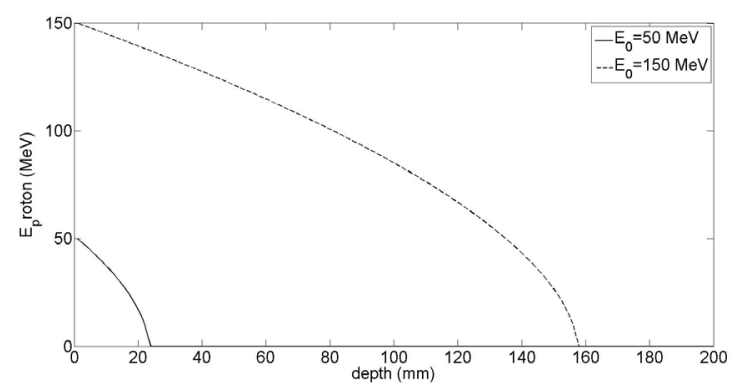

Fig 2. Protons energy as a function of depth in water for 50 $\mathrm{MeV}$ (solid line) and $150 \mathrm{MeV}$ (dashed line) $\mathrm{E}_{0}$.

\subsection{Spread-out Bragg peak}

It is well known that a pristine Bragg peak is not wide enough to cover most treatment volumes and therefore unsuitable for cancer treatment. Rather, it is necessary to "spread out" the Bragg peak to deliver uniform dose within the target volume, by providing a suitably weighted energy distribution of the incident beam. For that purpose poly-energetic pencil beams are utilized, which are individually adapted to the proximal and distal edge of the target volume, such that the dose is constant along the depth of the target volume. This creates a highly conformal high dose region, e.g., created by a spread-out Bragg peak (SOBP) with the possibility of covering the tumor volume with high accuracy. At the same time this technique delivers lower doses to healthy tissue than conventional photon or electron techniques. The mathematical problem consists of the calculation of weighting factors $W(R)$ for the Bragg peaks such that the superposition results in a flat SOBP of height $D_{0}$ within an interval $\left[d_{a}, d_{b}\right]$. Bortfeld et al. [17] have derived an analytical formulation of the weights $\mathrm{W}(\mathrm{R})$ as follows:

$W(R)=\left\{\begin{array}{l}\rho D_{0} \frac{p \sin (\pi / p) a^{1 / p}}{\pi\left(d_{b}-R_{0}\right)^{1 / p}}, \quad d_{a} \leq R_{0}<d_{b} \\ 0, \quad R_{0}<d_{a}, R_{0}>d_{b}\end{array}\right.$

The shape of the SOBP curve can now be calculated by estimating the $\mathrm{D}(\mathrm{z})$ (eq. 7) and $\mathrm{W}(\mathrm{R})$. In the special case of $p=1.5$ and $r<10$, the depth dose of the SOBP, $\mathrm{D}_{\mathrm{SOBP}}(\mathrm{r})$ can be approximated by a simpler relationship:

$$
D_{S O B P}(r) \approx \frac{D_{0}}{1+0.44 r^{0.6}}
$$

Deviations of eq. 9 are within $\pm 1.5 \%$ of $\mathrm{D}_{0}$. Figs. 3 and 4 illustrate a set of weighted Bragg peaks and their superposition creating a SOBP respectively.

\subsection{Lateral profiles of pencil beams}

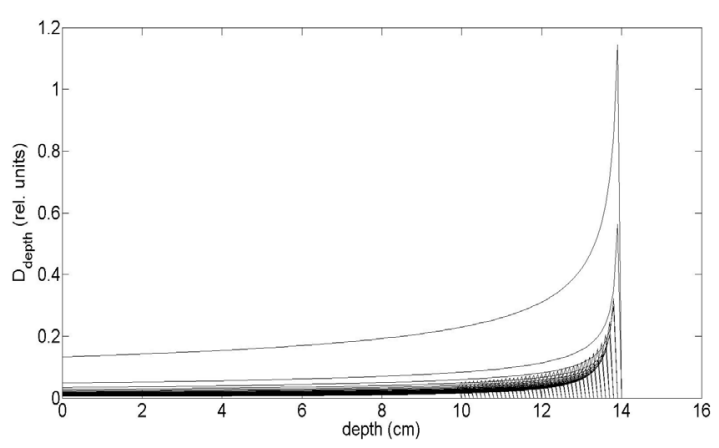

Fig. 3. A set of weighted pristine Bragg peaks of different energies.

Protons are assumed to emanate axial symmetrically from the finite-sized source with a specified mean residual range and with no angular emittance initially. For simplicity we assumed scattering only in the patient, which is increasing the radial emittance at the depth of 
the point of interest. The dose at a point of interest is then determined, by the residual range of protons directed at that point. This is equal to the residual range of protons entering the patient, minus the radiographic path length from the surface to the point of interest, $\mathrm{r}\left(\mathrm{x}_{\mathrm{p}}, \mathrm{y}_{\mathrm{p}}, \mathrm{z}_{\mathrm{p}}\right)$. The radiographic path length from the surface to the point of interest, $\mathrm{rpl}_{\mathrm{p}}$ is based on a pixelby-pixel integration through a CT study, given by:

$$
r p l_{p}=\int_{\text {surface }}^{z p} d z^{\prime} W E D\left(C T\left(z^{\prime}\right)\right)
$$

where $\mathrm{CT}\left(\mathrm{z}^{\prime}\right)$ is the CT value at the point of distance $\mathrm{z}^{\prime}$ along the path of integration, and WED is value from a look-up Table which converts between CT value and water-equivalent density, as described by Chen at al. [18]. To compute the dose at a point of interest from a given pencil beam, we follow the approach of Hogstrom et al. [19] who reported on a pencil beam algorithm to model electron beams. The dose, $\mathrm{d}\left(\mathrm{x}^{\prime}, \mathrm{y}^{\prime}, \mathrm{z}^{\prime}\right)$, at a point $\left(x^{\prime}, y^{\prime}, z^{\prime}\right)$, due to pencil beam is separated into a central-axis term, $\mathrm{C}\left(\mathrm{z}^{\prime}\right)$, and an off-axis term, $\mathrm{O}\left(\mathrm{x}^{\prime}, \mathrm{y}^{\prime}, \mathrm{z}^{\prime}\right)$ :

$$
d\left(x^{\prime}, y^{\prime}, z^{\prime}\right)=C\left(z^{\prime}\right) \cdot O\left(x^{\prime}, y^{\prime}, z^{\prime}\right)
$$

The central-axis term is taken from the broad-beam central-axis depth dose in water modified by an inverse square correction:

$$
C\left(z^{\prime}\right)=D\left(d_{e f f}\right) \cdot\left(\frac{s s d+d_{e f f}}{z^{\prime}}\right)^{2}
$$

Where $\mathrm{D}\left(\mathrm{d}_{\text {eff }}\right)$ is described in eq. 7 , ssd is the source-tosurface distance and $d_{\text {eff }}$ is the effective depth given by:

$$
d_{\text {eff }}=R_{0}-\left(R_{r}-\operatorname{rpl}\left(z^{\prime}\right)\right)
$$

The off-axis term $\mathrm{O}\left(\mathrm{z}^{\prime}\right)$ is taken to be the lateral flux distribution from the radial emittance suffered by protons directed along the axis of the pencil beam. The distribution is considered Gaussian [20]:

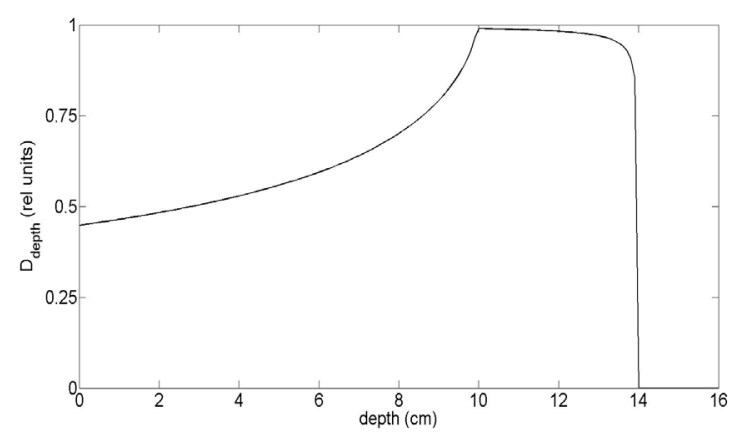

Fig. 4. Superposition of weighted Bragg peaks creating a SOBP.

$$
O\left(x^{\prime}, y^{\prime}, z^{\prime}\right)=\frac{\exp \left(-\left(x^{\prime 2}+y^{\prime 2}\right) / 2 \sigma\left(z^{\prime}\right)^{2}\right)}{2 \pi \sigma\left(z^{\prime}\right)^{2}}
$$

where :

$$
\frac{\sigma\left(z^{\prime}\right)}{\sigma\left(R_{0}\right)}=a_{1} \frac{z^{\prime}}{R_{0}}+a_{2} \frac{z^{2}}{R_{0}^{2}}
$$

and,

$$
\sigma\left(R_{0}\right)=0.02275 R_{0}+0.12085 \cdot 10^{-4} \cdot R_{0}^{2}
$$

Fig. 5 depicts plots of eq. 14 for $150 \mathrm{MeV}$ monoenergetic protons beam for various depths. We notice the increased variance, which reflects to larger penumbra of the $\mathrm{PB}$, as the depth increases.

\subsection{GPU-based parallelization}

The pencil beam method can be used to represent an incident broad beam as a collection of infinitesimally narrow pencil beams (PBs). The major assumptions in a PB dose calculation algorithm include: (1) the broad beam from a point source can be divided into identical beamlets, and (2) the total dose to a point $\mathrm{P}(\mathrm{x}, \mathrm{y}, \mathrm{z})$ is equivalent to integration over pencil beam dose contributions to $\mathrm{P}(\mathrm{x}, \mathrm{y}, \mathrm{z})$. A total dose distribution is obtained by a superposition of the contributions from elementary pencil beams. The total dose $\mathrm{D}(\mathrm{x}, \mathrm{y}, \mathrm{z})$ at point $(\mathrm{x}, \mathrm{y}, \mathrm{z})$ is calculated as follows:

$$
D(x, y, z)=\iint d x^{\prime} d y^{\prime} C^{\left(x^{\prime}, y^{\prime}\right)}(z) O\left(x-x^{\prime}, y-y^{\prime}, z\right)
$$

$C^{\left(x^{\prime}, y^{\prime}\right)}(z)$ indicates the central-axis term of the beam passing through a point $\left(\mathrm{x}^{\prime}, \mathrm{y}^{\prime}, \mathrm{z}\right)$. The second term, $\mathrm{O}\left(\mathrm{x}-\mathrm{x}^{\prime}, \mathrm{y}^{\prime} \mathrm{y}^{\prime}, \mathrm{z}\right)$, is obtained from eq. 14. In practice, the integration of eq. 17 is discretized. A region of interest is divided into small elements, which are called voxels. The voxel size is 1 or $2 \mathrm{~mm}$ in general. The discretization of equation (17) leads to the following expression for the dose at voxel $(\mathrm{i}, \mathrm{j}, \mathrm{k})$ :

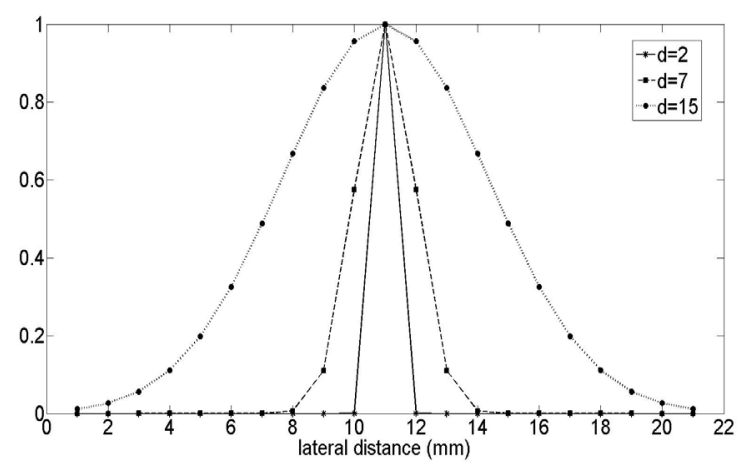

Fig. 5. Lateral distributions as a function of distance from the central axis for various depths $\mathrm{d}$ in $\mathrm{cm}$. 


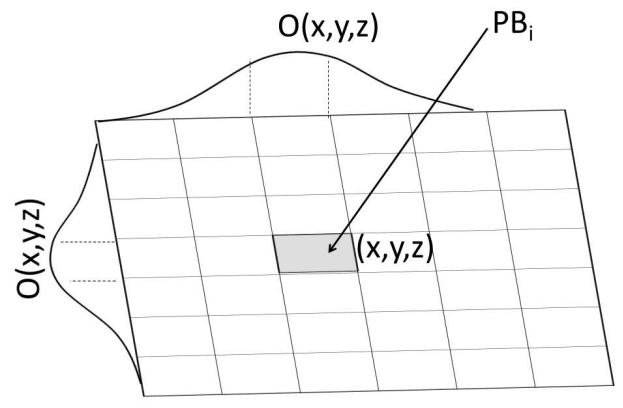

Fig. 6. Schematic representation of dose deposition into a particular voxel $(\mathrm{x}, \mathrm{y}, \mathrm{z})$ from the $\mathrm{PB}_{\mathrm{i}}$. The lateral Gaussianshaped curves represent the lateral penumbra of the $\mathrm{PB}_{\mathrm{i}}$.

$$
D(i, j, k)=\sum_{i^{\prime}, j^{\prime}} C^{\left(i^{\prime}, j^{\prime}\right)}(k) w\left(i^{\prime}, j^{\prime}, i, j\right)
$$

Where, w(i',j', i,j) indicates the dose contribution to a voxel $(\mathrm{i}, \mathrm{j})$ from the beam passing through a voxel $\left(i^{\prime}, j^{\prime}\right)$ within the k plane. Fig. 6 illustrates a graphical representation of the PB dose deposition method.

For the parallelization of the dose calculations, a native? approach would be that each thread in the GPU to handles a single PB and calculates the dose at adjacent points according to eq. 17. A different method would be, that each GPU in each thread concentrates on one particular calculation point (or else voxel) and accumulates the dose deposited by every single PB. The main disadvantage of the former method is the atomic operators, which are required in the case when more than one thread tries to write the deposited dose at the same voxel. In addition, for our case the code was implemented in MATLAB and we do not have access to each individual thread, rather algebraic operations of matrices are parallelized internally by the software by utilizing the high performance library CUBLAS. Therefore, we followed a different approach for the parallelization scheme. The dose calculations for the whole volume of each particular PB was parallelized on the GPU. That can be described by the following three steps:

(1) Calculate $\mathrm{C}(\mathrm{z})$ according to eq. 12 along each PB;

(2) Calculate $\mathrm{O}(\mathrm{x}, \mathrm{y}, \mathrm{z})$ according to eq. 14 along each beam path;

(3) Calculate the dose at each voxel according to eq. 17 .
Once the dose was calculated for a PB, the summation of the newly calculated dose at each voxel was also established on the GPU and the whole process is repeated for all the PBs.

\section{Results for HOMOGENEOUS PHANTOM}

\subsection{Materials and Elements}

In this section we introduce our computation environment and report our results of our method. The serial code was implemented in MATLAB and was launched on a desktop with a quad core Intel Xeon X5550 at $2.67 \mathrm{GHz}$ with $6 \mathrm{~GB}$ of RAM. For the parallelization on the GPU the parallel computing toolbox was employed and the code was launched on a GTX 770 with Kepler architecture, 1,536 CUDA cores grouped into eight SMs operating at $1046 \mathrm{MHz}$ and 2 GB of GDRR5 global memory. The performance comparison was established on the speedup factors as described by eq. 19 .

$$
\text { speedup }=\frac{T_{C P U}}{T_{G P U}}
$$

\subsection{Dose Distribution in Water Phantom}

Fig. 7 illustrates a 2D dose distribution of a $1 \mathrm{~mm}^{2}$ proton $\mathrm{PB}$ of $150 \mathrm{MeV}$ in water phantom. We notice the increased dose at the end of the particle trajectory due to the Bragg peak, as well as, the elevation of the off-axis dose as the depth increases. Isodose line distributions are shown in Fig. 8 for a $25 \times 25 \mathrm{~mm}^{2}$ proton beam of 50 $\mathrm{MeV}$. The $\mathrm{x}$-axis represents the depth of the beam in the water phantom, and the $y$-axis is the width of the open beam. Similarly we may observer the lateral spread of the beam as the depth increases. Finally, Fig. 9 depicts lateral profiles for a proton beam of $50 \mathrm{MeV}$ for four different depths. It is worth mentioning, that the units of $y$-axis are arbitrary units of the normalized dose to the $\max$.

\subsection{Evaluation of the algorithm performance}

The performance of the GPU code was evaluated for three different energies: low (50 MeV), medium (100 $\mathrm{MeV})$ and high $(150 \mathrm{MeV})$. Four square fields were selected for each energy, and the dose calculations were performed with both the serial and parallel codes for a homogeneous water phantom with size $300 \times 300 \times 300$ $\mathrm{mm}^{3}$. The resolution of the PBs was set to $1.0 \mathrm{~mm}$. Table I reports the details of the simulations we used in the current study. 
Table 1. Geometris used in performance evaluation

\begin{tabular}{ccrr}
\hline$E(\mathrm{MeV})$ & $P B S$ & $T_{C P U}(\mathrm{sec})$ & $T_{G P U}(\mathrm{sec})$ \\
\hline 50 & $25 \times 25$ & 50.8 & 1.1 \\
50 & $50 \times 50$ & 199.3 & 3.4 \\
50 & $80 \times 80$ & 515.6 & 8.5 \\
50 & $100 \times 100$ & 800.1 & 13.3 \\
100 & $25 \times 25$ & 115.0 & 1.4 \\
100 & $50 \times 50$ & 461.9 & 4.8 \\
100 & $80 \times 80$ & $1.16 \times 10^{3}$ & 11.6 \\
100 & $100 \times 100$ & $1.82 \times 10^{3}$ & 18.3 \\
150 & $25 \times 25$ & 208.4 & 1.8 \\
150 & $50 \times 50$ & 827.5 & 6.7 \\
150 & $80 \times 80$ & $2.1 \times 10^{3}$ & 16.7 \\
150 & $100 \times 100$ & $3.3 \times 10^{3}$ & 25.9
\end{tabular}

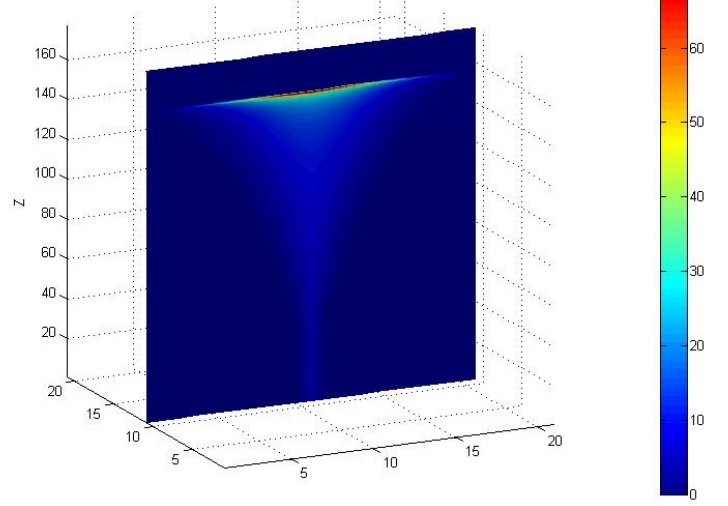

Fig. 7. 2D dose distribution of a proton PB with energy 150 $\mathrm{MeV}$ in water.

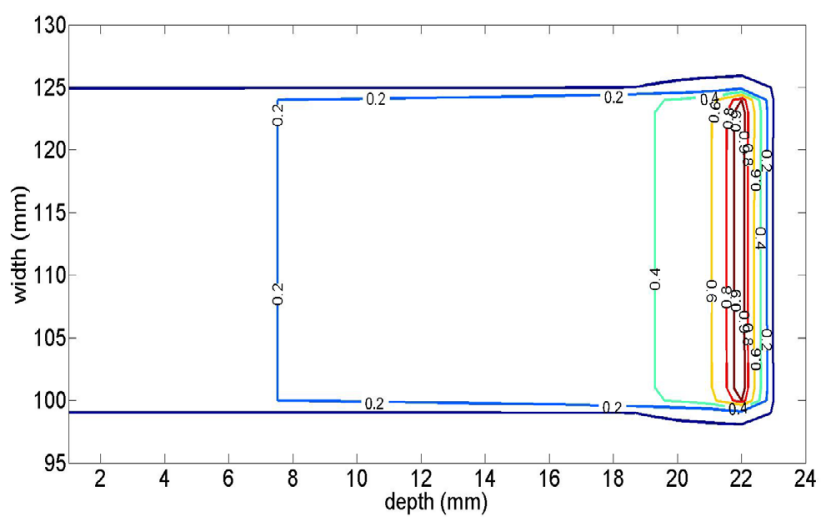

Fig. 8. Isodose distribution for a $25 \times 25 \mathrm{~mm}^{2}$ proton beam of $50 \mathrm{MeV}$ in water.

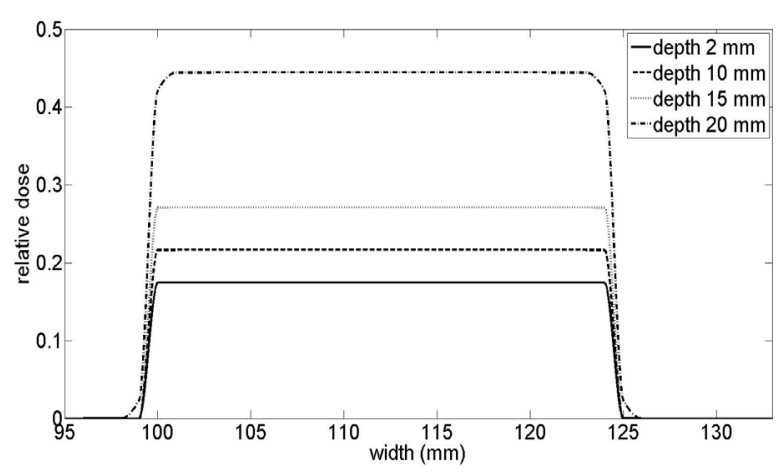

Fig. 9. Lateral profiles for a $25 \times 25 \mathrm{~mm}^{2}$ proton beam of 50 $\mathrm{MeV}$ in water at depth $2,10,15$ and $20 \mathrm{~mm}$.

Fig. 10 illustrates the speedup factors obtained from our simulation results. We notice that the speedup is decreased for smaller number of PBs and smaller energies. We speculate that this is due to the communication overhead between the GPU and the CPU during the iterations of the dose calculations for each PB. The maximum speedup of $\sim 127$ was achieved for the highest energy and the largest field size.

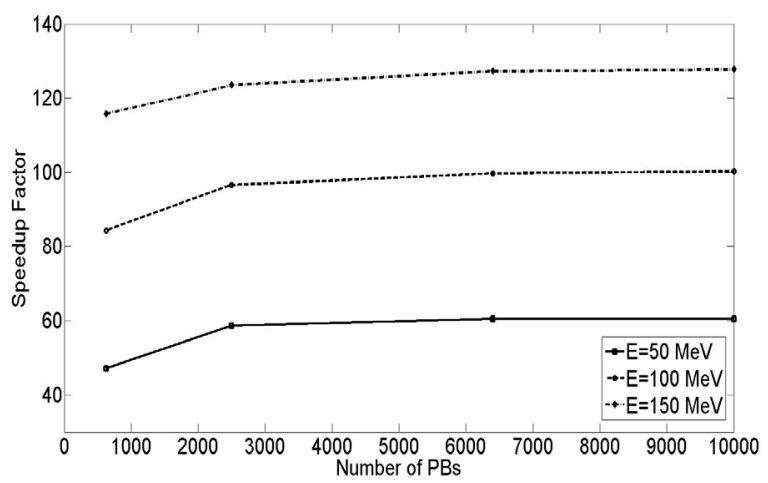

Fig. 10. Speedup factors as a function of energy and number of PBs

\section{Conclusion}

A GPU-based PB algorithm for proton dose calculations in Matlab was presented. A maximum speedup of $\sim 127$ was achieved. Future directions of the current work include extension of our method for dose calculation in heterogeneous phantoms. Work along this line is in progress. 


\section{References}

[1] R. R. Wilson, "Radiological use of fast protons," Radiology, vol. 47, pp. 487-91, Nov 1946.

[2] J. H. Lawrence, C. A. Tobias, J. L. Born, C. R. Mc, J. E. Roberts, H. O. Anger, B. V. Low-Beer, and C. B. Huggins, "Pituitary irradiation with high-energy proton beams: a preliminary report," Cancer Res, vol. 18, pp. 121-34, Feb 1958.

[3] A. M. Koehler and W. M. Preston, "Protons in radiation therapy. Comparative dose distributions for protons, photons, and electrons," Radiology, vol. 104, pp. 191-5, Jul 1972.

[4] A. J. Lomax, T. Boehringer, A. Coray, E. Egger, G. Goitein, M. Grossmann, P. Juelke, S. Lin, E. Pedroni, B. Rohrer, W. Roser, B. Rossi, B. Siegenthaler, O. Stadelmann, H. Stauble, C. Vetter, and L. Wisser, "Intensity modulated proton therapy: a clinical example," Med Phys, vol. 28, pp. 317-24, Mar 2001.

[5] U. Oelfke and T. Bortfeld, "Inverse planning for photon and proton beams," Med Dosim, vol. 26, pp. 113-24, Summer 2001.

[6] H. Paganetti, H. Jiang, and A. Trofimov, "4D Monte Carlo simulation of proton beam scanning: modelling of variations in time and space to study the interplay between scanning pattern and time-dependent patient geometry," Phys Med Biol, vol. 50, pp. 983-90, Mar 7 2005.

[7] Y. Kang, X. Zhang, J. Y. Chang, H. Wang, X. Wei, Z. Liao, R. Komaki, J. D. Cox, P. A. Balter, H. Liu, X. R. Zhu, R. Mohan, and L. Dong, "4D Proton treatment planning strategy for mobile lung tumors," Int J Radiat Oncol Biol Phys, vol. 67, pp. 906-14, Mar 12007.

[8] R. Kohno, K. Hotta, S. Nishioka, K. Matsubara, R. Tansho, and T. Suzuki, "Clinical implementation of a GPU-based simplified Monte Carlo method for a treatment planning system of proton beam therapy," Phys Med Biol, vol. 56, pp. N287-94, Nov 212011.

[9] P. P. Yepes, D. Mirkovic, and P. J. Taddei, "A GPU implementation of a track-repeating algorithm for proton radiotherapy dose calculations," Phys Med Biol, vol. 55, pp. 7107-20, Dec 72010 .
[10] P. P. Yepes, T. Brannan, J. Huang, D. Mirkovic, W. D. Newhauser, P. J. Taddei, and U. Titt, "Application of a fast proton dose calculation algorithm to a thorax geometry," Radiat Meas, vol. 45, pp. 1367-1368, Dec 1 2010.

[11] X. Gu, D. Choi, C. Men, H. Pan, A. Majumdar, and S. B. Jiang, "GPU-based ultra-fast dose calculation using a finite size pencil beam model," Phys Med Biol, vol. 54, pp. 6287-97, Oct 212009.

[12] R. Fujimoto, T. Kurihara, and Y. Nagamine, "GPUbased fast pencil beam algorithm for proton therapy," Phys Med Biol, vol. 56, pp. 1319-28, Mar 72011.

[13] D. Sanchez-Parcerisa, M. Kondrla, A. Shaindlin, and A. Carabe, "FoCa: a modular treatment planning system for proton radiotherapy with research and educational purposes," Phys Med Biol, vol. 59, pp. 7341-60, Dec 7 2014.

[14] T. Bortfeld, "An analytical approximation of the Bragg curve for therapeutic proton beams," Med Phys, vol. 24, pp. 2024-33, Dec 1997.

[15] M. R. Raju, Heavy Particle Radiotherapy, 1980.

[16] M. Lee, A. Nahum, and S. Webb, "An empirical method to build up a model of proton dose distribution for a radiotherapy treatment planning package," Phys. Med. Biol., vol. 38, pp. 989-998, 1993.

[17] T. Bortfeld and W. Schlegel, "An analytical approximation of depth-dose distributions for therapeutic proton beams," Phys Med Biol, vol. 41, pp. 1331-9, Aug 1996.

[18] G. T. Chen, R. P. Singh, J. R. Castro, J. T. Lyman, and J. M. Quivey, "Treatment planning for heavy ion radiotherapy," Int J Radiat Oncol Biol Phys, vol. 5, pp. 1809-19, Oct 1979.

[19] K. R. Hogstrom, M. D. Mills, and P. R. Almond, "Electron beam dose calculations," Phys Med Biol, vol. 26, pp. 445-59, May 1981.

[20] L. Hong, M. Goitein, M. Bucciolini, R. Comiskey, B. Gottschalk, S. Rosenthal, C. Serago, and M. Urie, "A pencil beam algorithm for proton dose calculations," Phys Med Biol, vol. 41, pp. 1305-30, Aug 1996. 